\title{
Efectos de las políticas sanitarias sobre la contención del gasto sanitario en Chile
}

Influence of sanitary policies on health care cost containment in Chile

\author{
Planck Barahona-Urbina ${ }^{1}$, Fernando Rodríguez ${ }^{2}$ \\ 'Doctor en Economía, Profesor, Facultad de Ingeniería, Departamento de Industria y Negocios, Universidad de Atacama, Chile. \\ ${ }^{2}$ Doctor en Economía, Profesor departamento de economía aplicada, Universidad de Salamanca, España.
}

\begin{abstract}
Resumen
Introducción: La literatura económica propone diversos mecanismos de pago a proveedores, con el objetivo de conseguir incentivos en la contención del gasto sanitario. Objetivos: Analizar los fundamentos teóricos y efectividad de los instrumentos de pago, pago asociado a diagnóstico (PAD) y pago por prestaciones valoras (PPV), en la contención de costos implementados en Chile a lo largo de los últimas dos décadas, y evaluar cuantitativamente los efectos que han tenido dichos mecanismos de pago prospectivos recientemente creados sobre la utilización de los servicios hospitalarios de la Región Metropolitana (RM) y demás regiones del país. Diseño: Análisis econométrico y no paramétrico. Lugar: Chile. Materiales: Se utilizó datos del Ministerio de Salud de Chile (MINSAL) y del Fondo Nacional de salud (FONASA). Intervenciones: Aplicación de la metodología de series temporales, análisis envolvente de datos y modelo lógit multinomial para detectar si los mecanismos de pago han tenido el efecto deseado en la contención de costos. Principales medidas de resultados: Efectos de los mecanismos de pago a hospitales. Resultados: Los resultados han puesto de manifiesto que el segundo mecanismo de pago introducido, además de no generar incentivos en la reducción de las estancias promedio a nivel nacional tampoco ha generado niveles de eficiencia mas altos. Conclusiones: El primer mecanismo de pago PAD tiene un efecto positivo en la contención del uso de recurso. Por otro parte, en el segundo mecanismo de pago PPV no solo se utiliza más recursos, sino que además es posible que el sistema esté actuando de forma perversa dificultando la adopción de medidas que favorezcan la eficiencia.
\end{abstract}

Palabras clave: Mecanismo de pago prospectivo, Pago asociado a diagnostico, pago por prestaciones valoradas.

\section{Abstract}

Introduction: The literature on economics proposes different provider payment mechanisms at hospitals in order to obtain incentives in health care cost containment. Objectives: To analyze the theoretical foundations of implemented cost restraint instruments, payment associated to diagnosis (PAD), and payment for valued assistance (PFV) during the last two decades and their effectiveness on the Chilean sanitary system. Design: Econometric and non parametric analysis. Setting: Chile. Materials: Data from Chile's Ministry of Health and the National Health Fund was used. Interventions: Application of time series methodology, data envelopment analysis and multinomial logit model to detect whether payment mechanisms have had the desired effect on cost containment. Main outcome measures: Effects of hospital payment system. Results: Results have shown that the second payment system, in addition to not creating incentives in average stays reduction nationally it has neither generated higher levels of efficiency. Conclusions: The first payment system had a positive effect in curbing resources use. The second mechanism of payment generated neither incentives in the reduction of average stay in the country nor higher levels of efficiency. That is to say, not only more resources are used but also it is possible that the system is acting in a wicked way making difficult to take measures that favors efficiency.

Key words: Prospective payment, payment associated to diagnosis, payment for valued assistance.

An Fac med. 2011;72(3):197-204

\section{INTRODUCCIÓN}

Dentro de las reformas llevadas a cabo en el sistema sanitario chileno se ha establecido diversos mecanismos de pago a hospitales, con el objetivo de conseguir incentivos en la contención del gasto sanitario. En el año 1995, se introduce un nuevo mecanismo de pago denominado PAD (pago asociado a diagnóstico), de naturaleza prospectiva, y en el año 2001 se introduce, en vista de las nuevas exigencias del sistema, el pago por prestaciones valoradas (PPV), también de naturaleza prospectiva. Estas reformas llevadas a cabo han pretendido generar los incentivos adecuados en los proveedores para contener el gasto sanitario y se enmarcan dentro del proceso de cambio denominado reforma financiera.

En los últimos años, Chile ha experimentado un significativo aumento de sus gastos en salud. Actualmente, el gobierno ha aumentado la partida presupuestaria en salud hasta llegar en el año 2009 a 5,4\% del producto interno bruto (PIB). Las causas del crecimiento del gasto sanitario son muy diversas, aunque entre las principales puede citarse el progreso científico y técnico de la atención sanitaria y el aumento de la demanda de asistencia médica, estimulada por factores tan variados como la mayor cultura sanitaria de la población, mayor confianza en la medicina, 
niveles de vida más altos, el deseo de la sociedad actual de disfrutar de la vida de la mejor y más amplia forma posible y, sobre todo, por el envejecimiento progresivo de la población, que por sí mismo aumenta el consumo de medicamentos y de asistencia sanitaria. Las políticas públicas llevadas a cabo por el gobierno por controlar el gasto sanitario han generado un debate sobre política sanitaria que se ha mantenido a partir de la década de los años noventa. En este contexto, y con miras a mejorar la eficiencia del sistema, el gobierno ha optado por diseñar nuevos mecanismos de pago a proveedores hospitalarios, con el fin de contener el gasto sanitario por el lado de la oferta, mejorando los incentivos para el comportamiento eficiente.

Dentro de las reformas llevadas a cabo en el sistema sanitario chileno, se ha establecido diversos mecanismos de pago a hospitales con el objetivo de conseguir incentivos en la contención del gasto sanitario. En los inicios de la reforma (1979), se diseñó un sistema de pago retrospectivo a los hospitales públicos (pago por servicio). En este mecanismo de pago (denominado pago por prestaciones valoradas, FAP), cada prestación que realizaba el servicio de salud era clasificada y facturada a fin de mes al nivel central, y reembolsaba al servicio de salud el monto correspondiente de ese mes. Sin embargo, al ser un mecanismo de pago retrospectivo estimulaba una mayor calidad, pero no una administración eficiente de los recursos. Por lo tanto, este sistema de pago no generaba incentivos adecuados para contener el gasto, puesto que todas las prestaciones otorgadas eran reembolsadas. La literatura sobre los efectos de los mecanismos de pago por servicio es abundante en este sentido ${ }^{(1-6)}$.

Los problemas de incentivos que generaban los mecanismos de pago anterior llevaron a modificarlo. Se pensó, entonces, en implementar una modalidad de pago mixto, es decir una combinación de pago prospectivo y reembolso parcial de costes (con presupuesto acotado), que sirviera para generar en el proveedor los incentivos adecuados para el mantenimiento de la calidad de la prestación ${ }^{(7,8)}$. Es por ello que, en 1995, para la atención secundaria y terciaria se diseñó un sistema de pago prospectivo denominado pago asociado a diagnóstico (PAD) que, mediante la estandarización de un conjunto de prestaciones, permitió relacionar la asignación de recursos financieros a grupos de diagnósticos. Básicamente, el pago asociado a diagnóstico se refiere a un conjunto de prestaciones médicas (denominada canasta) asociadas a una determinada patología (por ejemplo, hernia inguinal, apendicitis, peritonitis, tumor maligno de estomago, entre otras), con sus respectivas frecuencias y costes. La construcción de la denominada 'canasta PAD' considera las prestaciones, como los días cama, exámenes y procedimientos, intervención quirúrgica, las frecuencias de uso. Los precios unitarios fueron elaborados según criterio experto, con revisión de fichas clínicas y estudios nacionales e internacionales que sirvieron como referente. En definitiva, el PAD, al ser un mecanismo de pago prospectivo, cualquier mejora en el proceso productivo que implique una baja en los costes es absorbido por el establecimiento, ya que el nivel central paga siempre el valor estandarizado ${ }^{(9)}$. Entre las principales ventajas de este mecanismo de pago se incluye que incentiva la eficiencia técnica en la atención al paciente, ya que el pago se asocia a la resolución de la patología ${ }^{(10)}$. Genera un incentivo a la calidad, por sobre la cantidad de prestaciones realizadas. El proveedor de salud debe buscar entonces la combinación óptima de recursos para lograr el objetivo deseado de mejorar al paciente. Otra de las ventajas del PAD es que incentiva la eficiencia económica, dado que si se resuelve la patología con menos acciones que las contenidas en la canasta promedio, el proveedor de salud obtiene un excedente financiero de libre disponibilidad. Sin embargo, este mecanismo de pago exige velar por la calidad de las prestaciones, ya que el prestador podría aumentar la eficiencia a costa de la calidad, con el objetivo de disponer de los excedentes financieros. Un ejemplo de esto es el incentivo a disminuir los días de estancia, con el consecuente riesgo de efectuar altas prematuras ${ }^{(11,12)}$. Por otro lado, cuando el proveedor es remunerado bajo el esquema de pagos por diagnósticos, el incentivo es más débil, en el sentido de que el proveedor puede utilizar la asimetría de información en su beneficio a la hora de darle más o menos peso a la dolencia, con el objetivo de recibir la retribución correspondiente a un grupo más costoso. Conjuntamente con el sistema de pago PAD se estableció el otro componente denominado pago prospectivo por prestaciones (PPP) (en su conjunto pago mixto PAD-PPP). La idea básica del segundo componente de pago mixto (PPP) es que en una situación donde un diagnóstico de ingreso derivase en otro tipo de prestaciones debido a algún tipo de complicación, se establece que el pago se realice en base al PPP, es decir un pago por la atención efectivamente realizada. Este sistema de pago se aplica también para diagnósticos para las cuales no existe un PAD preestablecido, pero bajo un sistema prospectivo, es decir se paga en base al presupuesto asignado con una cierta cantidad tope.

Con el objetivo de hacer más transparente y eficiente el gasto en el 2002, se diseñó el mecanismo de pago prospectivo por prestaciones valoradas (PPV). La característica de este mecanismo de pago es que el Fondo Nacional de Salud (FONASA) y cada servicio de salud firman un convenio de producción hospitalaria para el año, considerando su capacidad de oferta real y potencial para cada una de las líneas contempladas en el PPV. Si se observa una subproducción, esto da origen a un ajuste del financiamiento, reduciéndolo. Por el contrario, una sobreproducción implicaría una renegociación en el financiamiento. Este sistema obliga al hospital a no quedarse con los excedentes financieros. Respecto de la cuantificación de los precios, el equipo técnico de FONASA selecciona una prestación específica a ser valorada, definiendo los 
procedimientos asociados, y se estima el coste promedio o denominado 'arancel' FONASA. Entonces, el convenio señala la cantidad de actividad y el precio establecido. De esta manera, el PPV generaría incentivos para controlar que la provisión de ciertas prestaciones no sea reemplazada por otras de menor coste y tener así un excedente financiero. La similitud del mecanismo de pago PAD es que ambos tienen carácter prospectivo. Si embargo, la diferencia está en que el PAD es una canasta de prestaciones con sus respectivas frecuencias y costes y el PPV se paga por prestación asociado a cada programa. Más concretamente, el PAD se refiere a un mecanismo de pago que actúa mediante la confirmación de una canasta de prestaciones, con un precio promedio independiente del total de prestaciones otorgadas; y el PPV se refiere a una asignación presupuestaria, también de carácter prospectivo, en que es posible cuantificar prospectivamente el precio y la cantidad de prestaciones a otorgar mediante un convenio anual del FONASA con los servicios. De esta manera, el PPV generaría incentivos para controlar que la provisión de ciertas prestaciones no sea reemplazada por otras de menor coste y tener así un excedente financiero.

\section{MÉTODOS}

En nuestro análisis hemos utilizado datos del Ministerio de Salud de Chile (MINSAL) y el Fondo Nacional de Salud (FONASA). En la primera etapa del análisis usamos datos de actividad hospitalaria a nivel nacional, de las 12 regiones del país más la región metropolitana (RM), de los años 1980-2006, utilizando como variable Proxy del gasto la estancia promedio hospitalaria. La variable estancia promedio nos indicará si los diseños de pagos a hospitales han tenido el efecto deseado sobre la contención en el empleo de los recursos y la mejora de la eficiencia. El objetivo ha sido evaluar, por tanto, cuantitativamente los efectos que han tenido los mecanismos de pago asociado a diagnóstico (PAD) y pago por prestaciones valoradas (PPV), en el uso de recursos. Para ello hemos hecho uso de la metodología de series temporales de manera de determinar si los mecanismos de pago introducidos han cumplido con las expectativas de reducción de las estancias promedios. En el análisis hemos incorporado dos variables dummy, para recoger el efecto que han tenido los mecanismos de pago en el gasto público y si han sido significativas. Dichas variables cualitativas las hemos introducido en el año 1995, año en que se implementó el mecanismo de pago prospectivo PAD, y en el año 2001, año en que se implementó el sistema de pago prospectivo PPV. El modelo univariante resultante sería de la forma siguiente:

$$
y_{t}=\beta_{0}+\delta_{1} D_{1}+\delta_{2} D_{2}+\beta_{3} y_{t-1}+\varepsilon_{t}
$$

donde $y_{t-1}$ es la variable endógena retardada un período; $\beta_{0}, \delta_{1}, \delta_{2}$ y $\beta_{3}$ las constantes del modelo; $D_{1}$ y $D_{2}$ son la variables Dummy en los años 1995 y 2001, respectivamente; y $\varepsilon_{t}$ es el ruido blanco, con media cero, varianza constante e independiente en el tiempo.

En la segunda fase de nuestro estudio y una vez detectada la significatividad de los cambios en las estancias promedios, en esta segunda parte se llevó a cabo un análisis de eficiencia regional mediante la técnica análisis envolvente de datos (AED), para obtener datos que permitieran posteriormente evaluar la posible relación causa-efecto de las medidas de contención en el empleo de los recursos sobre la eficiencia. Puesto que los datos por regiones únicamente estaban disponibles desde 1997, el análisis debió limitarse forzosamente a la introducción del mecanismo de pago PPV. Para ello, se utilizó datos de actividad hospitalaria, esto es, como variables inputs médicos, enfermeras, matronas, camas disponibles; y como variable output, el egreso hospitalario.

Para evaluar la diferencia de eficiencia productiva entre unidades hemos usado el método análisis envolvente de datos (AED), un método de programación matemática que convierte múlti- ples inputs y outputs medidos en una sola suma de productividad eficiente. El AED está basado en la eficiencia relativa ${ }^{(13,14)}$. Es decir, estos autores flexibilizan el supuesto de AED en la cual se asumía rendimientos constantes de escala (DEA-CCR), permitiendo que la topología de rendimiento a escala se caracterice por una tecnología variable -constante, creciente o decreciente-. Por consiguiente, para la medición de la eficiencia técnica seguiremos el método técnica (AED) orientación inputs, asumiendo rendimientos constantes de escala, que nos ayudarán a construir lo que se denomina superficie envolvente, frontera eficiente o función de producción del conjunto de entidades que están siendo objeto de análisis. Aquellas entidades que determinan la envolvente la denominaremos entidades eficientes y permitirán la evaluación de la eficiencia relativa de cada una de las entidades. Una de las ventajas del AED es que, por ser una técnica no-paramétrica, no supone alguna forma funcional de la relación entre los inputs y los outputs ni supone una distribución de la ineficiencia ${ }^{(15)}$, requiriendo tan solo algunas propiedades formales como en el conjunto de producción, tales como convexidad y libre disposición de los inputs y outputs, que permitan la aplicación de las técnicas de programación lineal en que se basa. Formalmente, el cálculo del puntaje de productividad eficiente, según el modelo DEA-CCR (cálculo de la eficiencia técnica global), se efectúa solucionando el siguiente problema de programación lineal. Sea $\mathrm{N}$ el número de regiones a analizar, las cuales utilizan una cantidad determinada de $\mathrm{M}$ inputs para producir S outputs. Para la i-ésima región, se obtiene una matriz $x$ de tamaño $(\mathrm{Mx} 1)$ inputs y una matriz $y$ de tamaño (Sx1) outputs. Por lo tanto, la matriz $\mathrm{X}(\mathrm{MxN})$ inputs $\mathrm{y}$ la matriz Y $(\mathrm{SxN})$ outputs representan los datos para el total de regiones analizadas. La representación del modelo como un problema de optimización lineal, asumiendo rendimientos constantes de escala, puede ser expresada de la siguiente forma (modelo DEA-CCR): 


$$
\begin{aligned}
\text { Min }_{\phi, \lambda} \phi & \\
\text { S.a }-y_{i}+Y \lambda & \geq 0 \\
\phi x_{i}-X \lambda & \geq 0 \\
\lambda & \geq 0
\end{aligned}
$$

donde el término $\phi$ es un escalar que multiplica al vector de inputs y representa el factor que pondera los inputs de la región evaluada, $x_{i}$ refiere los inputs de la i-ésima región a ser evaluada, $y_{i}$ representa los outputs de la i-ésima región a ser evaluada, $\mathrm{X}$ es la matriz de inputs $\mathrm{MxN}, \mathrm{Y}$ es una matriz de outputs $\mathrm{SxN}, \lambda$ es un vector de constantes $\mathrm{Nx} 1$ que multiplica a la matriz $\mathrm{X}$ e $\mathrm{Y}$-que describe la importancia de las regiones tomadas en consideración para determinar el producto virtual o unidad de referencia, y que sirve de comparación para evaluar la i-ésima región-; por último, $\mathrm{N}$ es el número de regiones. Debemos decir que el problema dual permite ilustrar acerca de la naturaleza de la eficiencia relativa, dado que se obtiene, en el caso de que existan, las holguras (slacks) o reducciones no radiales de inputs ${ }^{(16)}$. Para que una unidad sea eficiente, $\phi$ será igual a 1 y las holguras serán igual a 0 , esto es, la unidad observada se encontrará produciendo en la frontera óptima de producción. Con el fin de calcular la eficiencia técnica pura, modificamos el planteamiento anterior para incluir la posibilidad de rendimientos variables de escala:

$\begin{aligned} \text { Min }_{\phi, \lambda} \phi & \\ \text { S.a }-y_{i}+Y \lambda & \geq 0 \\ \phi x_{i}-X \lambda & \geq 0 \\ N 1^{\prime} \lambda & =1 \\ \lambda & \geq 0\end{aligned}$

En este modelo se incluye una restricción de convexidad (N1' $\lambda=1)$, donde $\mathrm{N} 1$ es vector unitario $\mathrm{Nx} 1$. Esta modificación permite descomponer la eficiencia en dos: por un lado, la eficiencia técnica pura (ETP), calculada bajo el método BCC, y por otro lado la eficiencia de escala, que es el resultado del cociente de los coeficientes de eficiencia calculados con el modelo CCR y el modelo BCC. Cabe señalar que, si existen diferencias entre las dos mediciones de eficiencia para una unidad (en nuestro caso regiones), entonces significa que dicha región posee ineficiencia de escala. Formalmente, podemos determinar residualmente la eficiencia a escala como:

$$
\operatorname{EE}\left(x_{i}, y_{i}\right)=\frac{\operatorname{ETG}\left(x_{i}, y_{i}\right)}{\operatorname{ETP}\left(x_{i}, y_{i}\right)}
$$

donde ETG es la eficiencia técnica global calculada mediante el método DEA-CCR, ETP calculada mediante el método DEA-BCC; $x_{i}$ e $y_{i}$ son los inputs de la unidad $i$ y los outputs de la unidad $i$, respectivamente.

En la fase anterior hemos previsto una serie de variables con posibilidad de situar a las regiones en distintos niveles de eficiencia. Esto da paso a un análisis de segunda etapa, basado en la utilización de una regresión en la que se incluye los índices de eficiencia iniciales $\left(\theta_{i}\right)$ como variables dependientes y las variables exógenas $\left(Z_{i}\right)$ como regresoras:

$$
\theta_{i}=f\left(Z_{i}, \beta_{i}\right)+u_{j}
$$

donde $\beta_{i}$ son los parámetros a estimar y $u_{j}$ es el ruido blanco, con media cero, varianza constante e independiente en el tiempo. Para responder por los factores que más elevan la probabilidad de las regiones de situarse en los grupos más eficientes plantearemos un modelo de regresión lógit multinomial, en el cual consideraremos como variable dependiente (endógena) la probabilidad de que el índice de eficiencia técnica pertenezca a los denominados nivel eficiente (CE), primero, segundo, tercero y cuarto grupo. Como base tomaremos el grupo uno y el grupo tres. Para una región con un vector de variables (variables exógenas), el modelo podemos expresarlo como:

$$
\begin{gathered}
P_{j} \equiv \operatorname{Pr}\left(\underline{C_{J}}<\phi<\overline{C_{J}}\right)=\frac{e^{\beta_{j}^{\prime} x_{i}}}{1+\sum_{k \in J} e^{\beta_{k} x_{i}}} \\
\operatorname{Con} j=J \equiv\{C E, 1,2,3\}
\end{gathered}
$$

donde $C_{J}$ y $\bar{C}_{J}$ simbolizan, respectivamente, los limites inferiores y superiores del cuartil j-ésimo, $\phi$ es el índice de eficiencia proporcionado por AED. Puesto que los datos por regiones únicamente están disponibles desde 1997, el análisis debe limitarse forzosamente a la introducción del mecanismo de pago PPV. Como variables de control

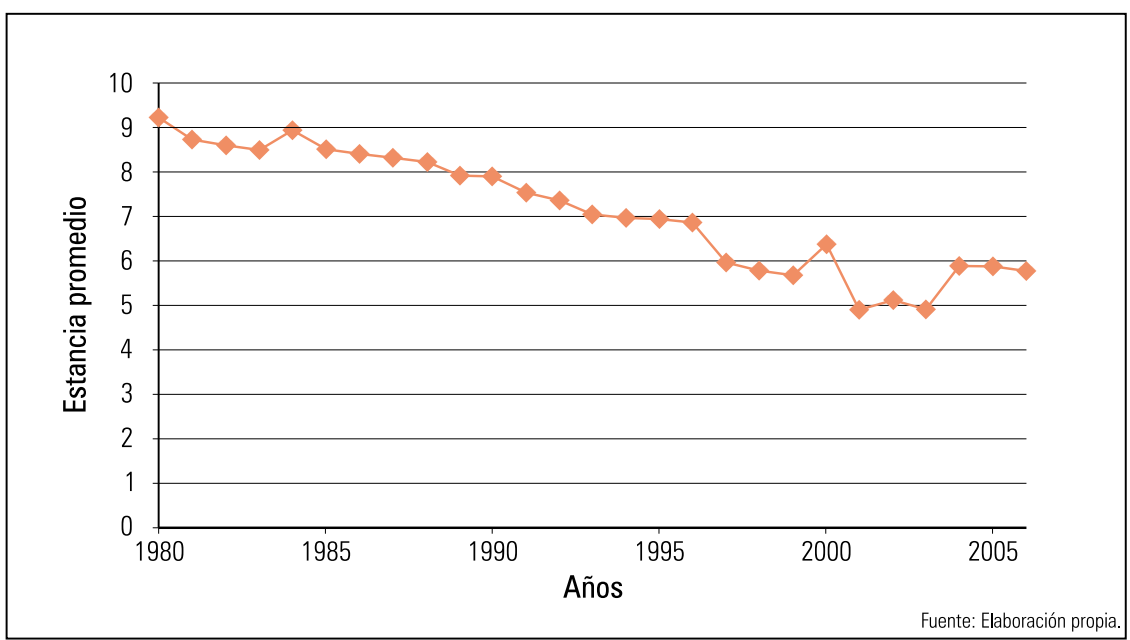

Figura1. Serie temporal, 1980-2006. Estancia hospitalaria promedio. 
Tabla 1. Modelo ARIMA ${ }^{(1,2)}$.

\begin{tabular}{|c|c|c|c|c|}
\hline \multicolumn{5}{|c|}{ Variable dependiente: Estancia promedio } \\
\hline Variables & Coeficiente & Error estándar & t-statistic & Prob. \\
\hline C & $-0,157414$ & 0,016857 & $-9,338214$ & 0,0000 \\
\hline D1 & $-0,141558$ & 0,048485 & $-2,919619$ & 0,0085 \\
\hline D2 & 0,289598 & 0,053370 & 5,426210 & 0,0000 \\
\hline$A R(1)$ & $-0,976396$ & 0,070301 & $-13,88886$ & 0,0000 \\
\hline $\mathrm{MA}(2)$ & $-0,929930$ & 0,074925 & $-12,41145$ & 0,0000 \\
\hline R-squared & 0,604761 & & & \\
\hline R-squared ajustada & 0,525713 & & & \\
\hline Prob (F-statistic) & 0,000656 & & & \\
\hline
\end{tabular}

Fuente. Resultado programa estadistica E-view 4.0.

hemos utilizado el PIB regional, superficie en $\mathrm{km}^{2}$ y población mayores de 65 años, y tratamos de ver el efecto del segundo mecanismo de pago. Para el lógit hemos distribuido los score de eficiencia calculado en el AED en grupos de menor a mayor eficiencia, tomando como referencia uno de esos grupos. Es decir, analizaremos la relación entre los mecanismos de pago introducidos y la probabilidad de que ello pueda haber influido en el nivel de eficiencia de los hospitales de las distintas regiones del país, mediante la aplicación de un modelo lógit multinomial.

\section{RESULTADOS}

Observemos que la serie temporal estancia promedio y su tendencia nos sugiere presencia de no estacionariedad (figura 1).
Dadas esta características y luego de hechas las transformaciones adecuadas y estacionarizada la serie, aceptamos el modelo ARIMA ${ }^{(1,2)}$ (tabla 1). Observemos que los coeficientes del modelo son todos significativos, por lo que, satisfacen las condiciones de estacionariedad e invertibilidad. Obsérvese también que el valor de la significatividad conjunta de los parámetros del modelo, dado a través del p-valor, es muy pequeña $(0.000656)$, lo que indica la aceptación de la hipótesis de la significatividad conjunta de todos los parámetros del modelo. La significatividad individual de cada parámetro se analiza a través de los parámetros p-valores de la columna prob, observándose que todos los parámetros estimados son fuertemente significativos individualmente (distintos de cero), por tener $\mathrm{p}$-valores muy pequeño.

Por lo tanto, no hay ningún obstáculo para aceptar el modelo:

$$
\begin{aligned}
\Delta Y_{t}= & -0.15-0.14 D_{1}+0.28 D_{2} \\
& 0.97 Y_{t-1}-0.92 \alpha_{t-2}+\varepsilon_{t}
\end{aligned}
$$

Tabla 2. Rendimiento a escala constante [AED-CCR]. Orientación inputs.

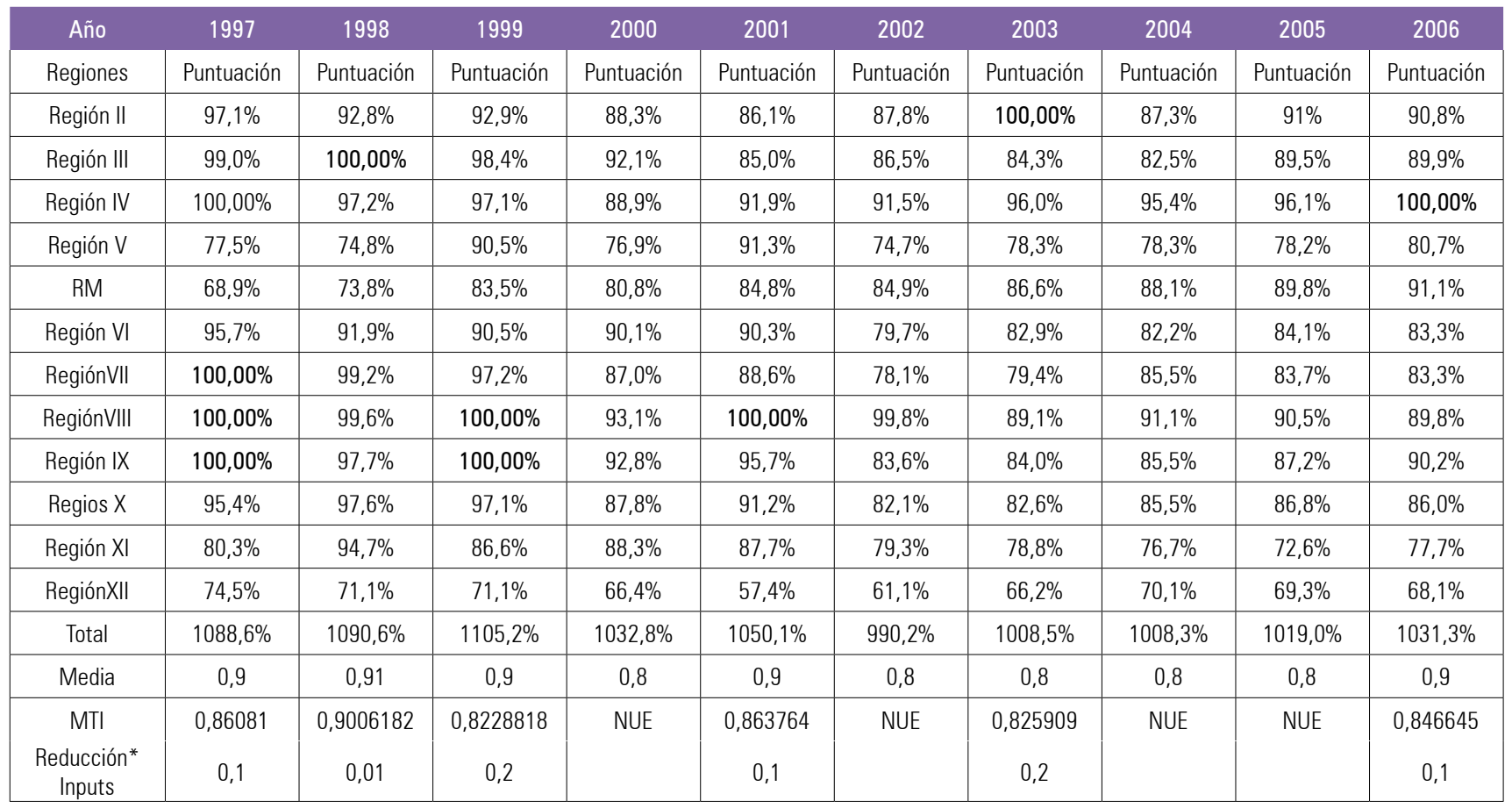

Fuente: Elaboración propia, a partir de datos proporcionados por el programa EMS (efficiency measurement system). 
Según esta ecuación, la introducción del mecanismo de pago en el año 1995 tuvo el efecto deseado, es decir, hubo una disminución de las estancias hospitalarias promedio. Sin embargo, la introducción del segundo mecanismo de pago al parecer no cumplió con las expectativas (signo positivo) a nivel global, sino que incluso se produce un cambio en la tendencia y se registra un incremento de las estancias hospitalarias promedio. Ello puede deberse a distintas circunstancias puntuales, aunque no hay cambios significativos en el sistema sanitario que permitieran predecir este resultado; en cualquier caso, el resultado pone de manifiesto la falta de efectividad del mecanismo de pago PPV para contener el uso de recursos.

En la segunda etapa, para el análisis AED, hemos utilizado como variables inputs determinadas categorías del personal sanitario -médicos, enfermeras y matronas-y de capital instalado - camas disponibles-, y como variable output el egreso hospitalario de las 11 regiones del país más la región metropolita (RM), del año 1997 hasta el año 2006. Hemos dejado forzosamente de lado el análisis para el año 1995 (introducción del primer mecanismo de pago), por carecer de datos. El objetivo era determinar cuáles de las regiones eran técnicamente más eficientes y obtener datos que permitieran posteriormente evaluar la posible relación causa-efecto de las medidas de contención en el empleo de los recursos sobre la eficiencia Los resultados del análisis envolvente de datos los observamos en la tabla 2. En este análisis se identifica las regiones técnicamente eficientes y aquellas que han quedado por debajo de la frontera eficiente. Ello nos ha permitido saber cuál de las regiones está haciendo un mejor uso de sus recursos. Aquí se observa las tendencias de la eficiencia relativa de las regiones a lo largo de toda la serie. Por ejemplo, en el año 97, las regiones técnicamente eficientes fueron las regiones 7, 8 y 9; las demás quedaron bajo la frontera eficiente, lo cual

Tabla 3. Resultados proporcionados por metodología lógit multinomial.

\begin{tabular}{|c|c|c|c|}
\hline & Variables & Coeficiente & $P>z$ \\
\hline \multicolumn{4}{|l|}{ C. Eficiente } \\
\hline & Superficie & $-4,13 \mathrm{E}-05^{* *}$ & 0,021 \\
\hline & Población > 65 años & $-0,000031$ & 0,146 \\
\hline & $\mathrm{PIB}$ & 0,2195308 & 0,266 \\
\hline & Dummy & $-1,316565$ & 0,114 \\
\hline & Constante & $3,302539 * *$ & 0,05 \\
\hline \multicolumn{4}{|l|}{ C4 } \\
\hline & Superficie & $-4,79 \mathrm{E}-05^{* *}$ & 0,001 \\
\hline & Población > 65 años & $-4,61 \mathrm{E}-05^{*}$ & 0,009 \\
\hline & PIB & $0,3101631^{*}$ & 0,066 \\
\hline & Dummy & $-1,455379 * *$ & 0,023 \\
\hline & Constante & $5,26849 * *$ & 0,000 \\
\hline \multicolumn{4}{|l|}{ C3 } \\
\hline & Superficie & $-0,0000224^{*}$ & 0,064 \\
\hline & Población > 65 años & $-0,0000186$ & 0,181 \\
\hline & $\mathrm{PIB}$ & 0,1855546 & 0,171 \\
\hline & Dummy & 0,5860623 & 0,355 \\
\hline & Constante & 1,417799 & 0,234 \\
\hline \multicolumn{4}{|l|}{ C2 } \\
\hline & Superficie & $-0,0000505^{* *}$ & 0,000 \\
\hline & Población > 65 años & $-0,0000444^{* *}$ & 0,004 \\
\hline & $\mathrm{PIB}$ & $0,425855^{* *}$ & 0,004 \\
\hline & Dummy & $2,027512^{* *}$ & 0,019 \\
\hline & Constante & $2,458309^{*}$ & 0,056 \\
\hline \multicolumn{4}{|c|}{$\begin{array}{l}\text { Grupo } 1 \text { como base de comparación. } \\
* \text { Indica significación para } \alpha=0,10 \\
* * \text { indica significación para } \alpha=0,05\end{array}$} \\
\hline
\end{tabular}

Fuente: Elaboración propia. $\mathrm{CE}=$ Nivel eficiente. $\mathrm{PIB}=$ producto interno bruto.

significa que no están haciendo un uso eficiente de sus recursos. Un análisis global nos diría, por ejemplo que, en el año 2006, la ineficiencia técnica media fue de $84 \%$, lo que significa que para situarse en la frontera eficiente deberían haber reducido sus inputs en $15 \%$.

En la tercera fase del estudio analizamos, la relación entre los mecanismos de pago introducidos y la probabilidad de que ello pueda haber influido en el nivel de eficiencia de los hospitales de las distintas regiones del país, mediante la aplicación de un modelo lógit multinomial. Puesto que los datos por re- giones únicamente están disponibles desde 1997, el análisis debió limitarse forzosamente a la introducción del mecanismo de pago PPV. Como variables de control hemos utilizado el PIB regional, superficie en $\mathrm{km}^{2}$ y población mayor de 65 años, y tratamos de ver el efecto del segundo mecanismo de pago. Para el lógit hemos distribuido los score de eficiencia calculado en el AED en grupos de menor a mayor eficiencia, tomando como referencia uno de esos grupos. Los resultados ponen de manifiesto que, si tomamos como base de comparación el primer grupo, el más ineficiente, los resultados nos 
Tabla 4. Comparación con un nivel intermedio de eficiencia, mediante el análisis lógit multinomial.

\begin{tabular}{|c|c|c|c|}
\hline & Variables & Coeficiente & $\mathrm{P}>\mathrm{Z}$ \\
\hline \multirow[t]{6}{*}{ C.E } & & & \\
\hline & Superficie & $-0,0000189$ & 0,292 \\
\hline & Población > 65 años & $-0,0000124$ & 0,56 \\
\hline & PIB & 0,0339762 & 0,861 \\
\hline & Dummy & $-1,902627^{* *}$ & 0,026 \\
\hline & Constante & 1,88474 & 0,247 \\
\hline \multicolumn{4}{|l|}{ C 4} \\
\hline & Superficie & $-0,0000255^{*}$ & 0,069 \\
\hline & Población > 65 años & $-0,0000275$ & 0,118 \\
\hline & $\mathrm{PIB}$ & 0,1246086 & 0,451 \\
\hline & Dummy & $-2,041442^{* *}$ & 0,002 \\
\hline & Constante & $3,850691^{* *}$ & 0,004 \\
\hline \multicolumn{4}{|l|}{$\mathrm{C} 2$} \\
\hline & Superficie & $-0,0000281^{* *}$ & 0,028 \\
\hline & Población > 65 años & $-0,0000258^{*}$ & 0,058 \\
\hline & $\mathrm{PIB}$ & $0,2403004^{*}$ & 0,06 \\
\hline & Dummy & $1,44145^{*}$ & 0,091 \\
\hline & Constante & 1,04051 & 0,356 \\
\hline \multicolumn{4}{|l|}{ C1 } \\
\hline & Superficie & $0,0000224^{*}$ & 0,064 \\
\hline & Población > 65 años & 0,0000186 & 0,181 \\
\hline & $\mathrm{PIB}$ & $-0,1855546$ & 0,171 \\
\hline & Dummy & $-0,5860623$ & 0,355 \\
\hline & Constante & $-1,417799$ & 0,234 \\
\hline
\end{tabular}

permiten deducir que la introducción del PPV ha influido positivamente en un pequeño incremento del nivel de eficiencia de las regiones sanitarias, ya que el coeficiente de la variable dummy tiene signo positivo y significativo sobre la probabilidad de mejorar ligeramente la eficiencia para pasar al grupo 2, pero que el efecto desaparece (o incluso se vuelve negativo) para pasar a niveles de eficiencia más altos. La variable PIB influye positivamente sobre el incremento de la eficiencia, mientras que la superficie y la proporción de personas mayores de 65 años influyen negativamente (tabla 3). do cambios con la intención de corregir sus falencias. Las reformas apuntaron a incrementar el principio de eficiencia diseñando nuevos mecanismos de pago a hospitales, que se constituyeron como uno de los objetivos prioritarios del gobierno. Estos mecanismos de pago se basaron en los resultados principales de economía de la salud sobre eficiencia, contención de costes y calidad de las prestaciones sanitarias. En el año 1995 se introduce un mecanismo de pago de naturaleza prospectiva -pago asociado a diagnóstico- denominada canasta PAD. En el año 2001, y con el objetivo de mejorar la eficiencia del sistema, se introduce un nuevo mecanismo de pago, pago por prestaciones valoradas (PPV). Una vez analizados los fundamentos teóricos de los mecanismos de pago hemos procedido a evaluar cuantitativamente los efectos que han tenido los mecanismos de pago prospectivos 'pago asociado a diagnóstico y pago por prestaciones valoradas' sobre la contención en el empleo de los recursos. El análisis empírico de 'series temporales' muestra que el mecanismo de pago asociado a diagnóstico (PAD), introducido en el año 1995, tiene un efecto positivo en la contención del uso de recursos. Sin embargo, el mismo tipo de análisis de series temporales muestra que el segundo mecanismo, pago por prestaciones valoradas (PPV), introducido en el año 2001, no produce algún efecto positivo sobre la contención del uso de recursos. De hecho, los resultados muestran que hay un cambio en la tendencia, compatible con el incremento del uso de recursos tras la introducción del mecanismo 'pago por prestaciones valoradas', en contra de los objetivos buscados. En la segunda fase del análisis se creyó necesario indagar finalmente por la relación entre la introducción de los mecanismos de pago y la probabilidad de que ello influyera en el nivel de eficiencia de los hospitales públicos de las distintas regiones del país, para lo cual se llevó a cabo un análisis de eficiencia de estos centros mediante la técnica no paramétrica de análisis de 
envolvente de datos y se relacionó los índices relativos de eficiencia obtenidos con otros factores explicativos en un modelo de regresión logística multinomial. El análisis ha detectado que el segundo mecanismo de pago (PPV) se presenta como un factor probable de situar a las regiones en los grupos intermedios de eficiencia, pero que el efecto desaparece para situarlas en grupos de eficiencia más altos. Como conclusión general, podemos decir que el segundo mecanismo de pago introducido, pago por prestaciones valoradas, además de no generar incentivos en la reducción de las estancias promedio a nivel nacional tampoco ha generado niveles de eficiencia más altos. Es decir, no solo se utiliza más recursos, sino que además es posible que el sistema esté actuando de forma perversa, dificultando la adopción de medidas que favorezcan la eficiencia.

Creemos, luego de nuestra investigación, que es necesario profundizar en el análisis de estas circunstancias, sobre todo en lo relacionado con los resultados del análisis relacionados con el pago por prestaciones valoradas, básicamente para averiguar qué factores del contexto han podido producir estos resultados contrarios a los objetivos de la introducción del mecanismo de pago.

\section{AGRADECIMIENTOS}

En la elaboración de esta investigación agradecemos al Ministerio de Salud de Chile (MINSAL), en particular al Fondo Nacional de Salud (FONASA), por permitirnos acceder a su archivo histórico y bases de datos en versión electrónica.

\section{REFERENCIAS BIBLIOGRÁFICAS}

1. Epstein A, Beqq C, McNeil B. The use of ambulatory testing in prepaid and fee-for service group practices: relation to perceived profitability. N Engl J Med. 1986;314(17):1089-94.

2. Hickson G, Altemeier WA, Perrin JM. Physician reimbursement by salary or fee-for-service: effect on a physician's practice behavior in a randomized prospective study, Pediatrics. 1987;80(3):344-50.

3. Greenfield S, Nelson M, Zubkoff W, Manning W, Rogers W, Kravitz RL, Keller A, Tarlov AR, Ware JE Jr. Variations in resource utilization among medical specialties and systems of care: results of the medical outcomes study. JAMA. 1992;267(12):162430 .

4. Davison S, Manheim L, Werner, S, Hohlen M, Yudkowsky B, Fleming G. Prepayment with office based physicians in public funded programs: results from the Children's Medicaid Program. Pediatrics. 1992;89(4):761-70.

5. Rosenberg SN, Allen DR, Handte JS, Jackson TC, Leto L, Rodstein BM, Stratton SD, Westfall $G$, Yasser R. Effect of utilization review in a feefor-service health insurance plan, N Engl J Med. 1995;333(20):1326-30.

6. Shaughnessy P, Schlenker R, Hittle D. Home health care outcomes under capitated and fee-for-service payment. Health Care Financ Rev. 1994;16(1):187222.
7. Ellis R, McGuire T. Supply- side and demandside cost sharing health care. J Econ Perspect. 1986;7(4):135-51.

8. Ellis RP. Creaming, skimping and dumping: provider competition on the intensive and extensive margins. J Health Econ. 1998;17(5):537-55.

9. Lenz R. Pago asociado a diagnóstico: Breve reseña histórica. Cuadernos de Economia. 1995;95:105-12.

10. Rodríguez J, Tokman M. Resultados y rendimientos del gasto en el sector publico de salud de Chile 1990-1999, CEPAL, Santiago de Chile, Serie Financiamiento del Desarrollo, Nº; 2000.

11. Cluter D. Empirical evidence on hospital delivery under prospective payment. MIT, Mimeo, 1990.

12. Langa K. Cost-containment and interpayer treatment differences. University of Chicago, Mimeo, 1993.

13. Banker RD, Charnes A, Cooper W. Some models for estimating technical and scale efficiencies in data envelopment analysis. Management Science. 1984;30(9):1078-92.

14. Charnes A, Cooper WW, Rodhes E. Measuring the efficiency of decisions making units. Eur $\mathrm{J}$ Operat Res. 1978;2(6):429-44.

15. Farrell MJ. The measurement of productive efficiency. J Royal Statistical Soc, Series A., 1957;120(3):253-90.

16. Pulido A. Modelos econométricos, Madrid: Editorial Pirámide S.A. 1987.

Trabajo recibido el 27 de marzo de 2011 y aceptado para publicación el 20 de junio de 2011.

Correspondencia:

Dr. Planck Barahona-Urbina

Correo electrónico: planck.barahona@uda.cl 